\title{
HISTOPATHOLOGICAL AND ULTRASTRUCTURAL EVALUATION OF THE EFFECT OF ELECTRONIC CIGARETTE SMOKE ON THE SUBMANDIBULAR GLAND OF ALBINO RATS
}

\author{
Ahmed Nabil* and Mona El Deeb**
}

\begin{abstract}
Introduction: E-cigarettes (E-cigs) have received a considerable attention within the last years and are among the newest products for reduction of tobacco injury. Recently, it has been postulated that they are not considered a safe substitute for smoking and may have a limited degree of effectiveness in arresting smoking.
\end{abstract}

Aim: The aim of this study was to detect the histopathological and ultrastructural changes of tissue architecture of the submandibular glands in rats after passive E-cig exposure.

Methods: 12 adult male albino rats were divided into three groups (four rats each). Group I (Control group): Rats were exposed to fresh air. Group II (E-cigarette 4 weeks): Each rat was exposed to passive E-cig smoke for duration of one hour daily. The exposure time was divided into 6 equal intervals of $10 \mathrm{mins} / \mathrm{hr}, 7$ days a week for four successive weeks. Group III (E-cigarette 12 weeks): Rats of this group were exposed to the same dose and manner of treatment of E-cig smoke as in group II for 12 weeks duration. Animals were sacrificed following each treatment period and specimens were prepared for histological examination and transmission electron microscopic study.

Results: H \& E results revealed signs of atrophy and degeneration within the parenchymal elements together with dilatation and congestion of blood vessels with interstitial hemorrhage in 4 weeks E-cig exposed rats. More deleterious changes with marked distortion of the gland's architecture were detected in 12 weeks E-cig exposed group. These data were supported by the electron microscopic results which reflected degenerative changes in cytoplasmic organelles.

Conclusion: E-cig is not safe and its use to replace cigarette smoking cannot be recommended.

KEYWORDS: E-cigarettes, submandibular gland.

* Lecturer, Oral Pathology Department, Faculty of Dentistry, Beni Suif University.

** Associate Professor, Oral Biology Department, Faculty of Oral and Dental Medicine, Future University. 


\section{INTRODUCTION}

Electronic cigarettes (E-cig) are now considered as the most promising products for reduction of the deleterious effect of tobacco (Polosa et al., 2013) ${ }^{(\mathbf{1})}$. The device is electrically-driven, containing a battery part and an atomizer. The liquid is aerosolized by applying energy and generating heat to a resistance encircling a wick (Farsalinos and Polosa, 2014) ${ }^{(2)}$.

E-cigs are used recently to aid in quitting smoking. Interestingly, they are also marketed among never smokers (Manani et al, 2013) ${ }^{(3)}$. In contrast to conventional smoking, they can be freely used indoors and are considered to be safe environmentally (Henningfield and Zaatari, 2010) (4). It was also reported that chemicals produced during the combustion process in conventional smoking are absent in E- cigs. This indicates that E-cigs are thought to be healthy alternative to tobacco cigarettes (Farsalinos and Polosa, 2014) ${ }^{(2)}$.

On the other hand, Kim and Shin, 2013 (5) stated that the E-cig vapor consists of a mixture of propylene glycol and/or glycerol, nicotine and some added flavors. This vapor produces different toxic or carcinogenic chemicals such as particulates, formaldehyde, nitrosamines, metals, carbonyls, volatile organic compounds, and polycyclic aromatic hydrocarbons. Additionally, heat generation by the battery results in considerable raise of the level of these chemicals after vaporization (Kosmider et al, 2014) ${ }^{(6)}$. Moreover, the quality and safety of E-cig liquid (e-liquid) is not certainly insured (Scheffler et al, 2015) ${ }^{(7)}$.

Meanwhile, small particles emitted from the tobacco cigarette smoke are likewise produced from the E-cig vapor and may precipitate on the respiratory epithelium (Bertholon et al, 2013) ${ }^{(8)}$. They may also be able to induce carcinogenicity-related gene expression in human bronchial epithelial cells similarly to tobacco cigarettes (Cressey, 2014) ${ }^{(9)}$.
Moreover, the World Health Organization (WHO) has postulated that E-cigs are not considered a safe substitute for smoking. Recent studies indicate that E-cigs may have a limited degree of effectiveness in arresting smoking (Bertholon et al, 2013) ${ }^{(10)}$.

Obviously, restricted studies are available on effects of E-cigs in humans and in vivo animal models (Thomas et al, 2015) ${ }^{(11)}$.

Wu et al, $2014^{(12)}$ found that nicotine-free e-liquid promoted IL-6 production and human rhinovirus (HRV) infection in human airway epithelial cells. The effect of the nicotine-free eliquid was intensified by addition of nicotine, which proves the damaging effects of E-cigs in the airways of young people.

It was reported that passive smoking is related to various dental problems together with changes in salivary flow rate, decrease in salivary $\mathrm{pH}$, protein levels and buffering capacity (Avsar et al, 2008) ${ }^{(13)}$. The salivary glands are one of the tissues commonly subjected to smoke. Nevertheless, it is debatable whether or not passive cigarette exposure is correlated to noticeable changes in these tissues (Ferragut et al, 2011) ${ }^{(14)}$. The objective of this study was to observe the histopathological changes of tissue architecture of the submandibular glands in rats after passive E-cig exposure.

\section{MATERIAL \& METHODS}

12 adult male Albino rats of average weight 150-200 gm were used in this study. The animals were housed in separate hygienic cages under the optimal experimental conditions and fed on ground barely and supplied water add-libitum. They were kept in a clean well ventilated room according to the guidelines of the Animal Ethics Committee. The protocol of this study was approved by the Ethical Committee of $6^{\text {th }}$ of October University. The animals were randomly divided into three groups four rats each: 
Group I (Control group): Animals of this group were exposed to fresh air in the inhalation chamber instead of cigarette smoke.

Group II (E-cigarette 4 weeks): Rats of this group were exposed to passive E-cig* smoke in the inhalation chamber. Each rat was exposed to passive smoke for duration of one hour daily. The exposure time was divided into 6 equal intervals of $10 \mathrm{mins} / \mathrm{hr}, 7$ days a week. This was carried out for four successive weeks (OECD, 2009) ${ }^{(15)}$.

Group III (E-cigarette 12 weeks): Rats of this group were exposed to the same dose of passive E-cig smoke and were treated in the same manner as in group II for 12 weeks duration (OECD, 2009) ${ }^{(16)}$.

\section{Inhalation Chamber}

The mode of exposure was nose only exposure in the rate of one rat/experiment. The chamber's dimensions were $22 \times 12 \times 18 \mathrm{~cm}$ (OECD, 2009) ${ }^{(15,16)}$.

Following each treatment period, the animals were sacrificed by ketamine overdose. Samples of submandibular glands were gently dissected out and washed. Each specimen was divided into 2 halves to be prepared for routine histological examination and transmission electron microscopic study.

\section{Light microscopic study}

The first half was fixed in 10\% neutral formalin for 48 hours and embedded in paraffin. Histological sections of $5 \mu \mathrm{m}$ thickness were obtained and stained with haematoxylin and eosin stain.

\section{Transmission electron microscopic examination}

The second half was kept in $1.5 \%$ glutaraldehyde solution and $2 \%$ paraformaldehyde in phosphate buffer (PH. 7.4), and post fixed in osmium tetraoxide. The samples were then dehydrated in ascending grades of ethanol propylene oxide and embedded in epon. Ultrathin sections were stained with uranyl acetate and lead citrate and viewed with JEOL, JEM-100S electron, National Cancer Institute, Cairo University (Hayat, 1986) ${ }^{(17)}$.

\section{RESULTS}

\section{H\&E results}

\section{Control group (group I)}

Normal architecture of the submandibular salivary gland's elements was detected. The acinar cells were intermediate in structure between serous and mucous cells (seromucous). The acini were coalesced to each other exhibiting basal rounded nuclei and homogenous basophilic cytoplasm. The intervening striated ducts appeared intact with normal columnar cell lining (Fig. 1;a). The excretory ducts showed normal pseudostratified ductal outline within the connective tissue septa in-between the lobules of the gland (Fig. 1;b)

\section{E-cigarette 4 weeks (group II)}

Histological findings of this group revealed spacing between the acini. Signs of atrophy and degeneration could be detected among the gland's elements. Some acini appeared collapsed and shrunken; others showed indistinct acinar and cellular boundaries with numerous cytoplasmic vacuolation. Few nuclei appeared flattened, while some acini exhibited degenerated nuclei. The striated ducts appeared degenerated with indistinct ductal outlines and again revealed abnormal configuration. Extravasated blood was marked between the parenchymal elements (Fig. 2;a). The excretory duct showed some areas of degeneration of lining epithelium. Some epithelial cells were desquamated and appeared in clusters within the duct (fig. 2;b). Dilated and congested blood vessels with interstitial hemorrhage were obvious between the parenchymal tissues (fig. 2;c)

\footnotetext{
* Commercially available cigarettes (Like E-liquid Soprano, made in Italy) composition: propylene glycol, glycerol,
} nicotine, flavors and water. 


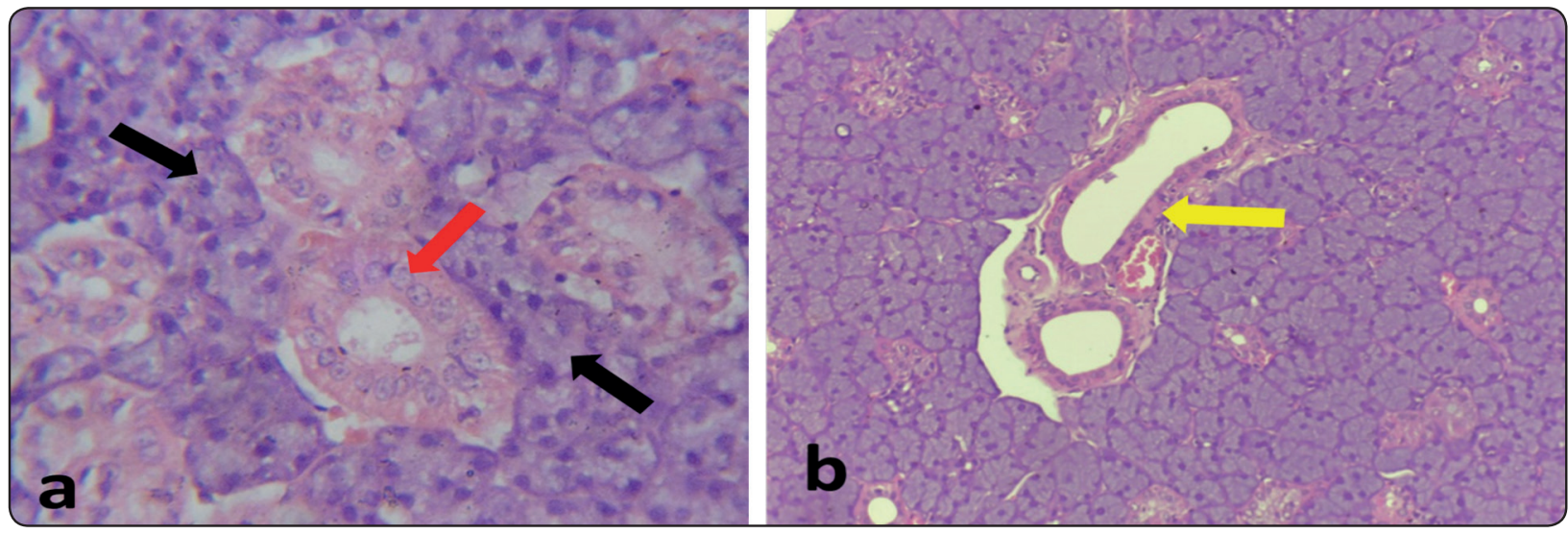

Fig. (1) Photomicrograph of rat submandibular salivary gland of group I showing: (a): seromucous acini (black arrows), striated duct (red arrow). (b): excretory duct (yellow arrow) (H\&E: (a) 400X, (b) 200X).
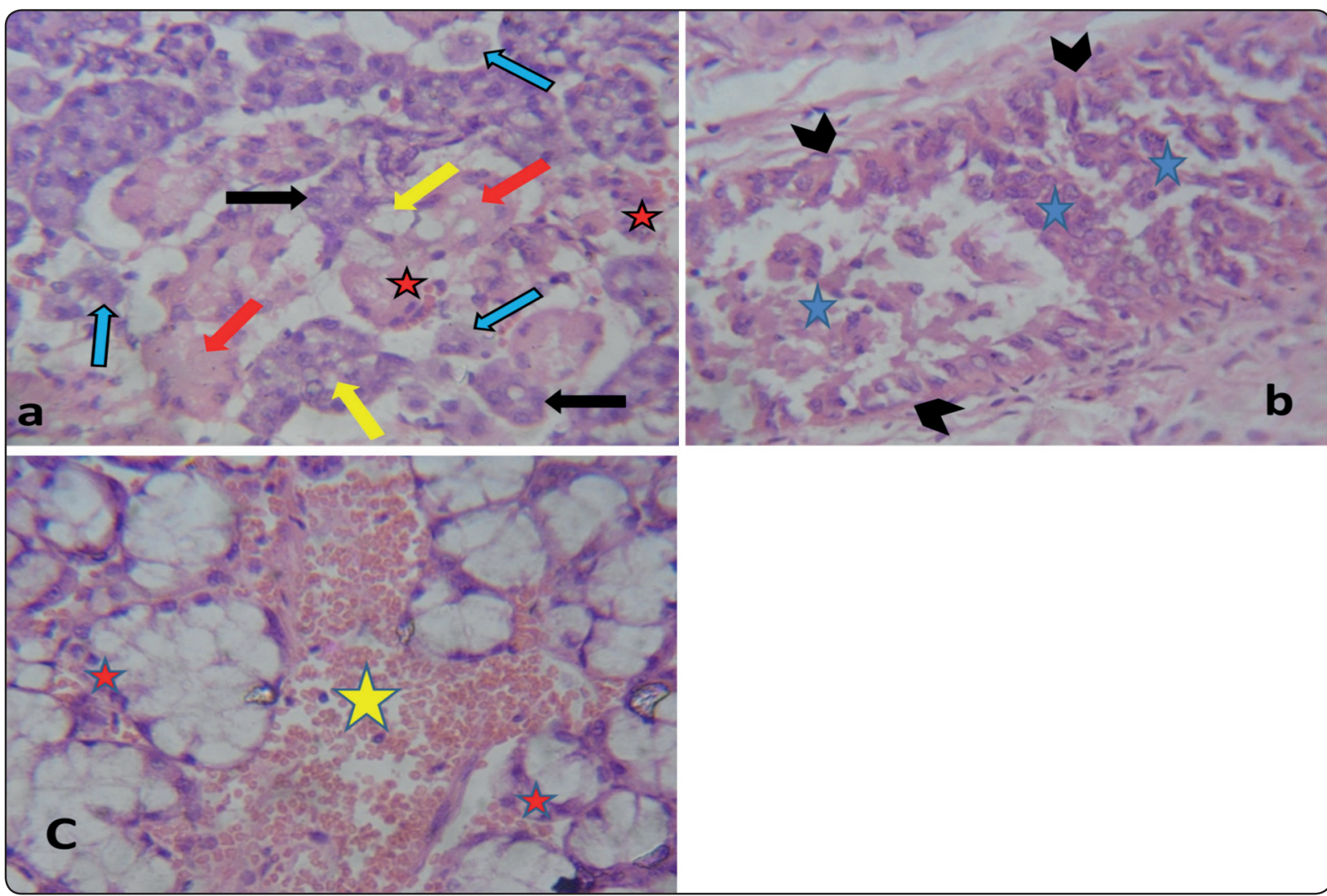

Fig. (2) Photomicrograph of rat submandibular salivary gland of group II showing: (a): collapsed acini \& indistinct acinar boundaries (black arrows), cytoplasmic vaculation (yellow arrows), degenerated acinar nuclei (blue arrows), degenerated striated ducts \& GCT (red arrows), Extravasated blood (red stars). (b): areas of degeneration of excretory duct epithelium (black arrow heads), desquamated epithelial cells (blue stars). (c): dilated and congested blood vessel (yellow star), interstitial hemorrhage (red stars) (H\&E: (a) 400X, (b) 400X, (c) 400X) 


\section{E-cigarette 12 weeks (group III)}

In comparison to the previous group, similar histological findings have been detected with more deleterious changes in the glandular parenchyma. Marked distortion of the gland's architecture with apparent extensive parenchymal spacing and widened connective tissue septa were obviously seen (fig. 3;a). Massive atrophy and degeneration of the acini and ducts could be detected with nuclear pleomrphism and disrupted ductal outlines (fig. 3;b). The excretory duct showed flattened, disfigured and atrophied epithelial lining with fragmented epithelial clusters inside (fig. 3;c). Moreover, the blood vessels were markedly congested and loaded with RBCs together with proliferated perivascular fibrosis (fig. 3;d).

\section{Transmission electron microscope results}

\section{Control group (group I)}

Parenchymal cells of the submandibular salivary gland showed normally appearing rounded nucleus with regular outline and homogenously distributed chromatin (Fig. 4). Active and well-developed rough endoplasmic reticulum was observed throughout the cytoplasm together with abundant mitochondria with clearly detected cristae (Figs. 5, 6). In addition, secretory granules (Fig. 4) and some lysosomes were also noted (Fig. 5)
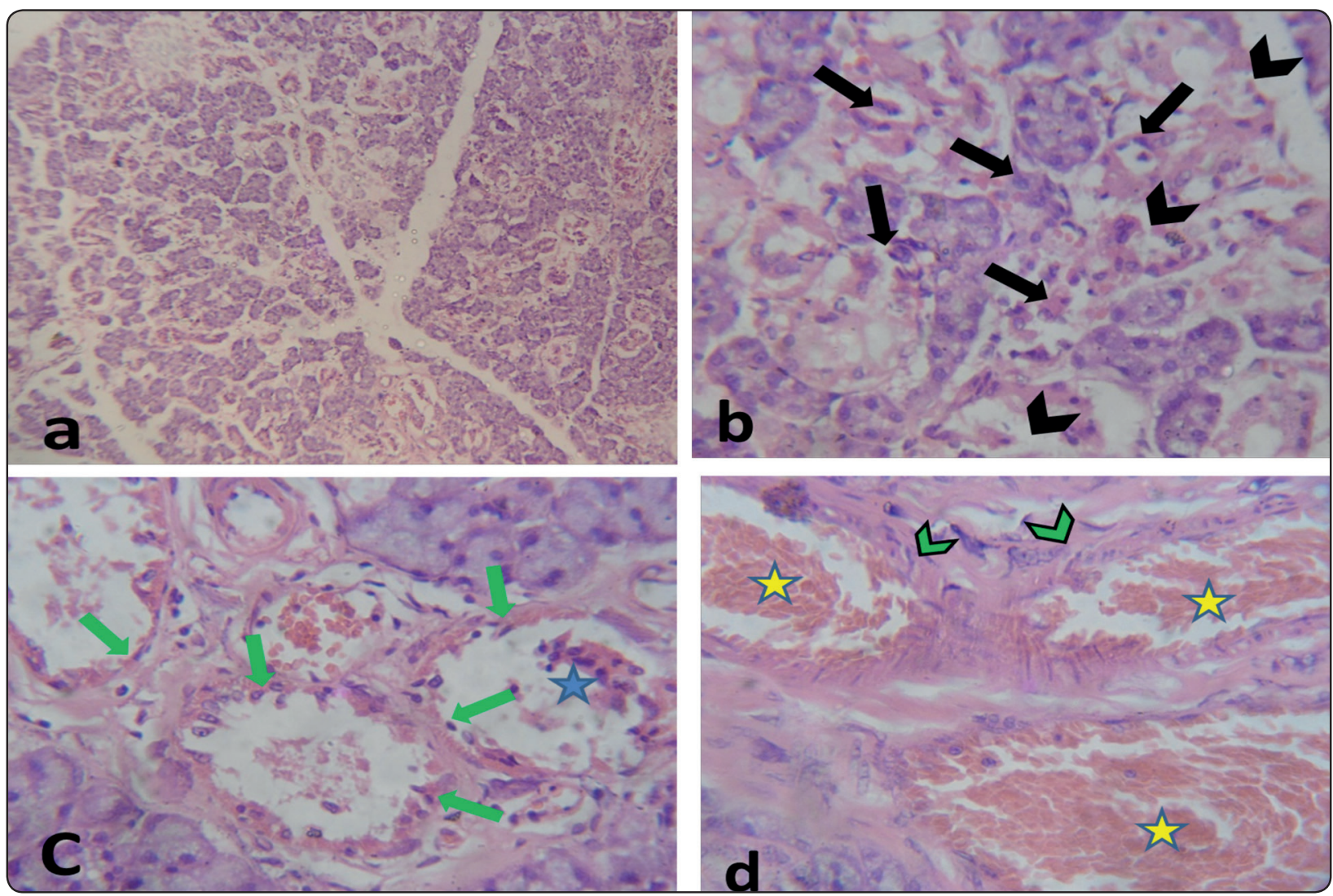

Fig. (3) Photomicrograph of rat submandibular salivary gland of group III showing: (a): extensive parenchymal spacing \& widened C.T. septa. (b): massive atrophy of the acini and ducts with nuclear pleomrphism (black arrows), disrupted ductal outlines (black arrow heads). (c): flattened \& disfigured epithelial lining (green arrows), fragmented epithelial clusters (blue star). (d): congested blood vessel (yellow stars), proliferated perivascular fibrosis (green arrow heads) (H\&E: (a) 200X, (b) 400X, (c) 400X, (d) 400X) 


\section{E-cigarette 4 weeks (group II)}

Under E/M, this group showed signs of cellular damage. The nucleus presented irregular nuclear membrane with peripheral and unevenly distributed chromatin. The cytoplasm showed signs of

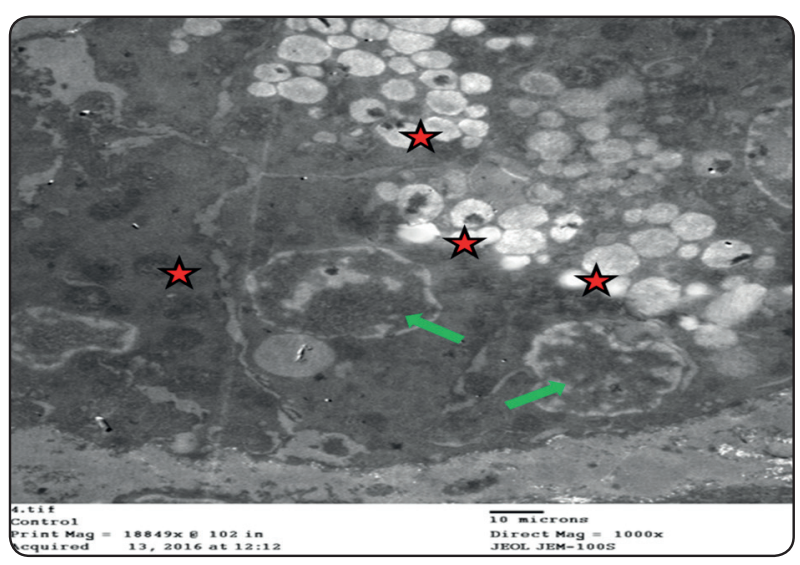

Fig. (4) Electron micrograph of seromucous cell of the submandibular gland acini group I showing: rounded nucleus and homogenously distributed chromatin (green arrows), secretory granules (red stars) (Uranyl acetate and lead citrate 1000X).

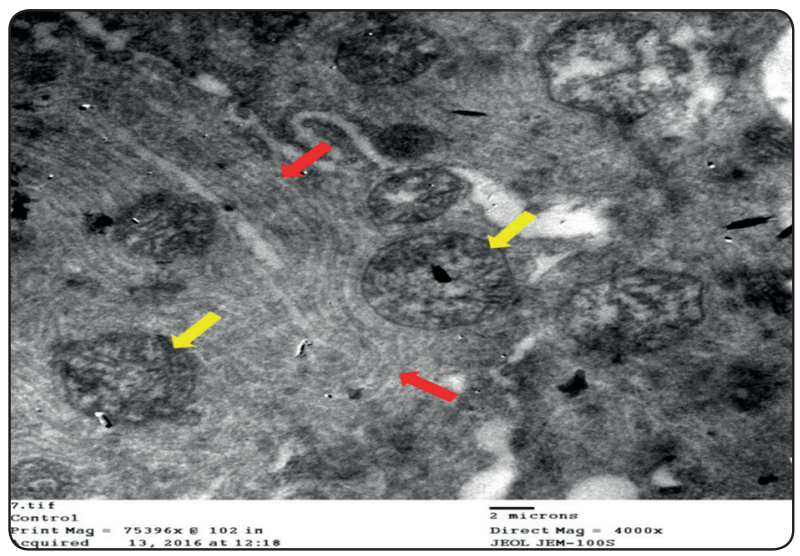

Fig. (6) Electron micrograph of seromucous cell of the submandibular gland acini group I showing: welldeveloped rough endoplasmic reticulum (red arrows), mitochondria with its cristae (yellow arrows) (Uranyl acetate and lead citrate 4000X). degeneration with intervening secretory granules forming pools of secretion (Fig. 7). Moreover, dilated sacs of rough endoplasmic reticulum were basally located. On the other hand, the mitochondria appeared moderately distended, pleomorphic with partially disrupted cristae (Figs. 7, 8).

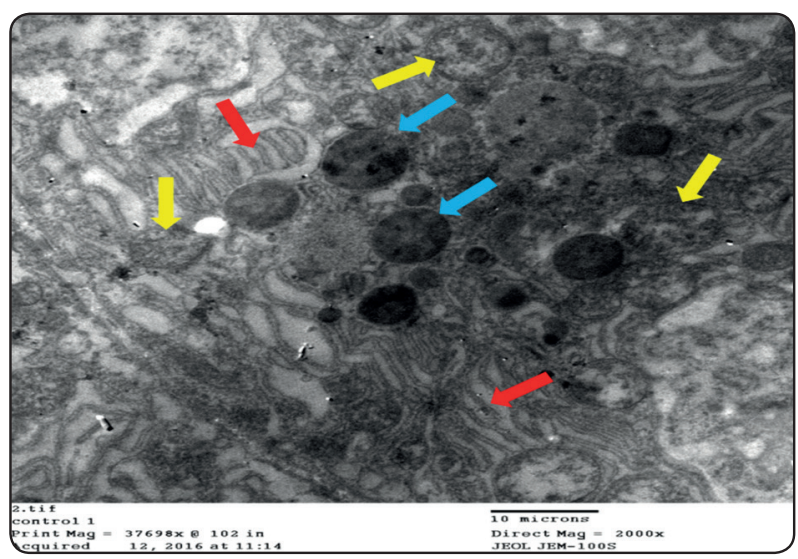

Fig. (5) Electron micrograph of seromucous cell of the submandibular gland acini group I showing: welldeveloped rough endoplasmic reticulum (red arrows), mitochondria (yellow arrows), lysosomes (blue arrows) (Uranyl acetate and lead citrate 2000X).

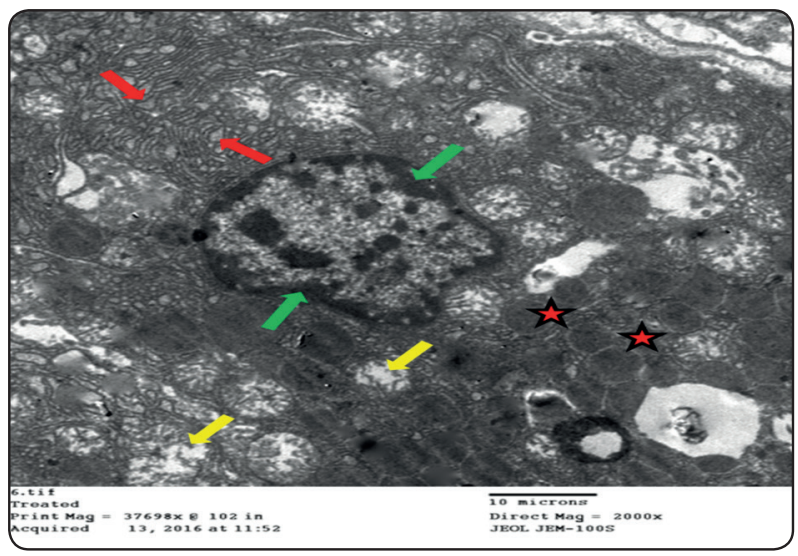

Fig. (7) Electron micrograph of seromucous cell of the submandibular gland acini group II showing: irregular nuclear membrane \& peripheral chromatin (green arrows), secretory granules (red stars), dilated sacs of rough endoplasmic reticulum (red arrows), mitochondria with partially disrupted cristae (yellow arrows) (Uranyl acetate and lead citrate 2000X). 


\section{E-cigarette 12 weeks (group III)}

Severe degenerative changes could be detected in 12 weeks E-cigarette smoke exposed group when compared to the previous group. Morphologically abnormal nuclei and alteration of the nuclear membrane were detectable features. Some nuclei exhibited areas of condensed, irregularly distributed chromatin together with irregularity

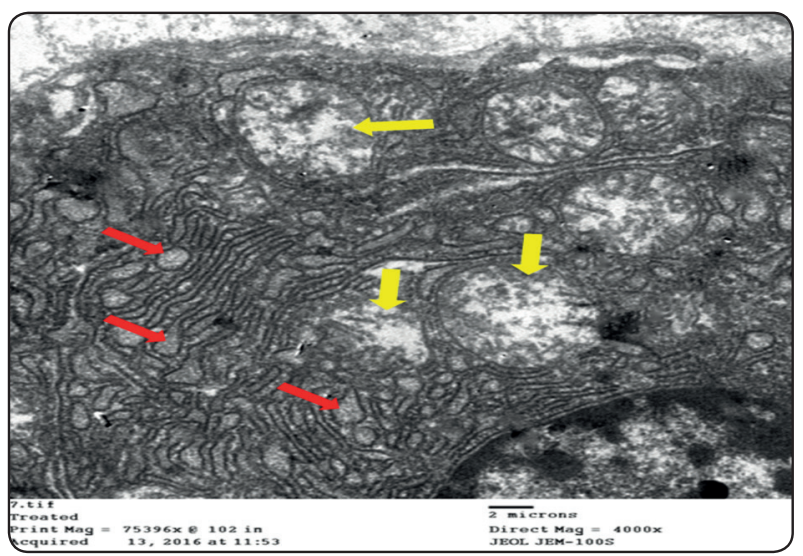

Fig. (8) Electron micrograph of seromucous cell of the submandibular gland acini group II showing: dilated sacs of rough endoplasmic reticulum (red arrows), distended mitochondria with partially disrupted cristae (yellow arrows) (Uranyl acetate and lead citrate 4000X).

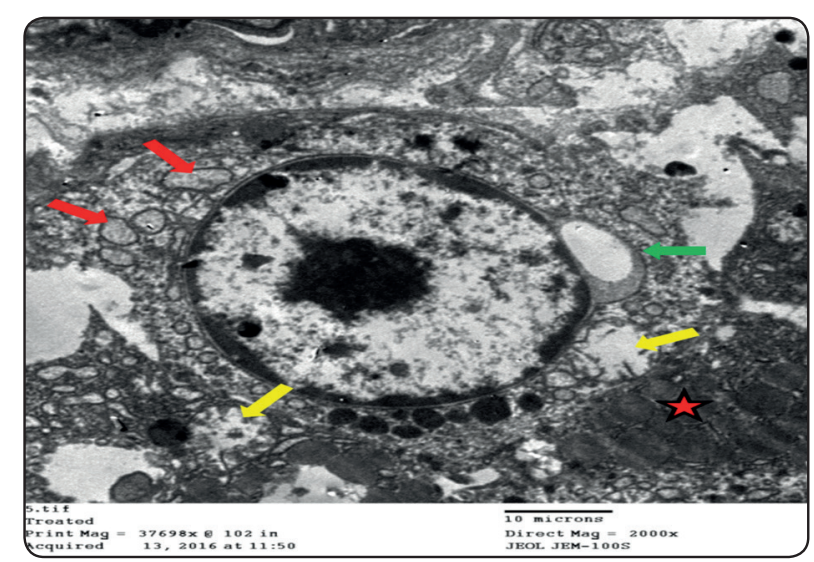

Fig. (10) Electron micrograph of seromucous cell of the submandibular gland acini group III showing: dilatation of the nuclear membrane (green arrow), secretory granules (red star), dilated and disrupted rough endoplasmic reticulum (red arrows), mitochondrial degeneration with disrupted cristae (yellow arrows) (Uranyl acetate and lead citrate 2000X). and dilatation of their nuclear membrane (Figs. 9, 10). Meanwhile, the cytoplasm showed massive vacuolar degeneration (Fig. 9) while the secretory granules were hardly detected (Fig. 10). The rough endoplasmic reticulum was sparse, disrupted, severely diminished and abnormally dilated. Some areas showed mitochondrial degeneration with disrupted or disappeared cristae (Fig. 10).

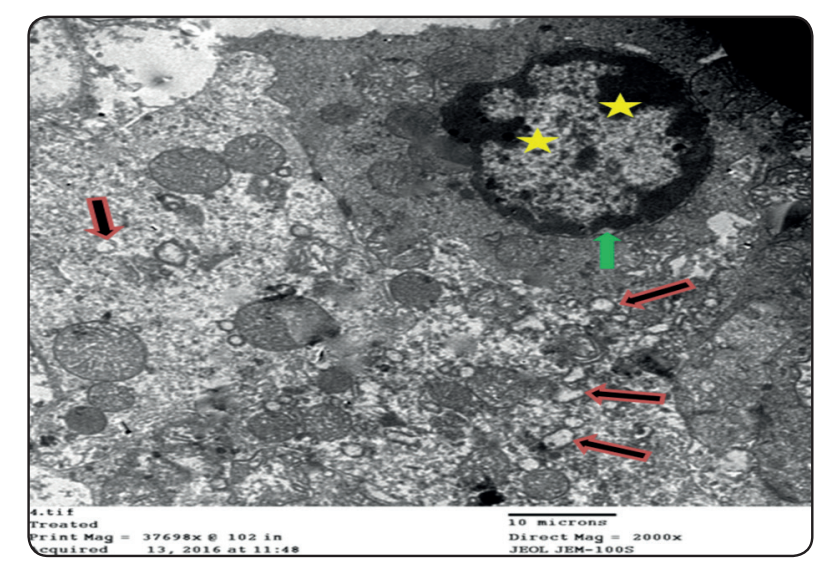

Fig. (9) Electron micrograph of seromucous cell of the submandibular gland acini group III showing: condensed, irregularly distributed chromatin (yellow stars), dilatation of the nuclear membrane (green arrow), vacuolar degeneration of cytoplasm (black arrows) (Uranyl acetate and lead citrate 2000X).

\section{DISCUSSION}

Smoking was found to induce various effects on the oral cavity and associated structures which predispose to precancerous and cancerous lesions. The salivary glands are one of the most important affected structures (Neville and Day, 2002) ${ }^{(18)}$. E-cigarettes have received a considerable attention and popularity within the last years. They play major role in nicotine replacement therapy (Ayers et al, 2011) ${ }^{(19)}$ and are among the newest and most hopeful products for reduction of tobacco injury (Polosa et al. 2013) ${ }^{(1)}$. Commercially sold E-cigs contain a wide range of nicotine concentrations (Etter, 2014) ${ }^{(20)}$. The level of nicotine exposure from use of E-cigs is markedly variable (Callahan-Lyon, 
2014) ${ }^{\text {(21) }}$. It is well known that nicotine is absorbed through the airway, skin, mucous membranes and gastrointestinal tract (Nowakowski, 1997) ${ }^{(22)}$. To date, limited data are available regarding the clinical effects of E-cig use, also nearly no established animal models of E-cig exposure is introduced (Thomas et al, 2015) ${ }^{(11)}$.

In the ongoing study, histological examination of submandibular salivary glands of E-cig treated groups revealed spacing between the acini and widening of the connective tissue spaces. Similar results were observed by Binsawad et al, $2011{ }^{(23)}$, who reported widening of intertubular spaces with a decrease in interstitial tissue elements of rats' testicular tissue exposed to cigarette smoke. A relevant study showed increased spaces between the prostatic acini in nicotine treated rats (Lina et al, 2014) ${ }^{(24)}$. Also, Valenca et al, $2008{ }^{(25)}$ suggested that nicotine induced toxic effects in liver resulting in reduction of hepatocytes number.

Major histopathological changes were noticed in group II treated rats and were severely expressed in group III, indicating signs of degeneration and deterioration. These changes included atrophy and collapse of the acini and ducts with indistinct boundaries. These forgoing observations are in agreement with Valenca et al, $2008{ }^{(25)}$ and Gawish et al, $2012{ }^{(26)}$ who verified cloudy cellular limits and histoarchitecture loss of hepatocytes of nicotine treated rats. Moreover, Cunha et al, 2010 (27) demonstrated de-epithelization of the circumvallate papillary surface and glandular atrophy in cigarette smokers. Endometrial glands also exhibited marked atrophy in rats subjected to smokeless tobacco (Singha, and Kazi, 2015) ${ }^{(28)}$. The toxicity of nicotine or its related metabolites may explain the observed degenerative and atrophic changes (Bao et al, 2002) ${ }^{(29)}$. Furthermore, it was found that E-cigs' vapor contain silicate particles and metal elements, which may have some cytotoxic effects (Williams et al, 2013) ${ }^{(30)}$.
On the other hand, some acini and ducts showed disappearance or flattening of their nuclei. This result was concomitant with El-Meligy et al, $2007^{(31)}$ and Iranloye and Bolarinwa, $2009^{(32)}$ in nicotine treated rats' ovary and heart cells respectively. Meanwhile, areas of cytoplasmic vacuolation apparent in groups II and III were parallel to that reported by El-Meligy et al, $2007^{(31)}$, Fujinami et al, $2009^{(33)}$ and Binsawad et al, $2011^{(23)}$ indicating degeneration and functional loss.

The previously mentioned degenerative changes could be explained by the existence of a significant amount of free radicals in E- cigs' vapor, which results in increase in oxidative stress. Although this amount of free radicals is lower than that produced from conventional cigarette smoke, it is considerable that could be potentially toxic to cells. Also, the battery output voltage plays a vital role in generation of other toxic compounds (Kosmider et al, 2014) (6). Moreover, it is conceivable to suggest that the histological damage caused by the administration of a nicotine source may be attributed to reduction in glutathione level and glutathione peroxidase activity (enzymes which protect from oxidative damage) (Neogy et al., 2008) ${ }^{(34)}$. The deficiency of antioxidant system is a result of an imbalance between the generation of reactive oxygen species (ROS) and antioxidant levels. When these species react with biomolecules in the cell, including proteins and nucleic acids, oxidative damage and destruction of cell membrane may occur and can lead to cells death (McCord, 1993) ${ }^{(35)}$ and (Aoshiba and Nagai, 2003) ${ }^{(36)}$. Recent study documented that human bronchial epithelial cells subjected to E-cigs' vapor show significantly lower cell viability as well as higher oxidative stress levels when compared to clean air exposed cells (Scheffler et al, 2015) ${ }^{(7)}$.

On the other hand, dilated and congested blood vessels with interstitial hemorrhage were noted between the parenchymal tissues in the treated groups. This data was coincident with Binsawad et al, $201{ }^{(23)}$ and Dhouib et al, $2014^{(37)}$ who 
identified dilated and engorged blood vessels with large number of RBCs in cigarette smoke and nicotine subjected rats' testicular and liver tissues respectively. In this regard, Dianzani, 1985 (38) proposed that nicotine can induce hematological changes in experimental animals which may indicate the enhanced erythropoiesis. Meanwhile, group III treated rats also showed proliferated perivascular fibrosis. This was in close agreement with Gawish et al, 2010 ${ }^{(39)}$ who observed thickened and hyalinizied wall of arterioles in the widened intertubular regions in testicular glands of nicotine treated mice.

Transmission electron microscopic results supported those of $\mathrm{H} \& \mathrm{E}$ stained results. Irregular nuclear membrane with peripheral and unevenly distributed chromatin was constant observations in E-cig smoke exposed groups. Signs of degeneration of intracellular organelles and cytoplasmic vacuolation were also documented. These results were strongly supported by Cervellati et al, $2014{ }^{(40)}$ who postulated that human lung epithelial cells exposed to E-cigs revealed increase in vacuolization, degeneration of organelles and expansion of the mitochondria and the endoplasmic reticulum. In another study, Binsawad et al, 2011 (23) verified ring shaped arrangement of peripheral chromatin in testicular tissue of rats exposed to cigarette smoke. On the basis of these available results, suggestions could be interpreted that ROS cause injury to the cell membrane resulting in leakage of intracellular materials and influx of calcium together with decrease in ATP (adenosine tri-phosphate) level and nuclear chromatin destruction. Increased ROS production also leads to diminished mitochondrial function and subsequently reduction of the energy supply in old cells (Toyokuni, 1999) ${ }^{(41)}$. Yamazaki et al, $1999{ }^{(42)}$ also stated that one of the mechanisms by which nicotine enhances generation of ROS is disrupting the mitochondrial respiratory chain.

A matter of concern is that all histopathological findings appeared to be more exaggerated with prolongation of the exposure period, which was markedly supported by other investigators (Binsawad et al, 2011) ${ }^{(23)}$ and (Gawish et al, 2012) ${ }^{(26)}$.

Based on the formerly mentioned data, it can be concluded that passive E-cig smoking led to deleterious structural changes in the submandibular salivary glands, which could significantly affect function with prolonged exposure. This indicates that E-cig is not really safe and its use to replace cigarette smoking cannot be recommended.

\section{REFERENCES}

1- Polosa, R., Rodu, B., Caponnetto, P., Maglia, M. and Raciti, C.: A fresh look at tobacco harm reduction: the case for the electronic cigarette. Harm Reduct. J., 10: 19, 2013 b.

2- Farsalinos, K. and Polosa, R.: Safety evaluation and risk assessment of electronic cigarettes as tobacco cigarette substitutes: a systematic review. Ther. Adv. Drug Saf., 5: 67-86, 2014.

3- Manani, M., Jegatheesan, P., DeSandre, G., Song, D., Showalter, L. and Govindaswami, B.: Elimination of Admission hypothermia in preterm very low-birth-weight infants by standardization of delivery room management. Perm.J., 17: 8-13, 2013.

4- Henningfield, J. and Zaatari, G.: Electronic nicotine delivery systems: emerging science foundation for policy. Tob. Control, 19: 89-90, 2010.

5- Kim, H. and Shin, H.: Determination of tobacco- specific nitrosamines in replacement liquids of electronic cigarettes by liquid chromatography-tandem mass spectrometry. J. Chromatogr. A.: 1291:48-55, 2013.

6- Kosmider, L., Sobczak,A., Fik, M., Knysak, J., Zaciera, M., Kurek, J. and Goienwicz, M.: Carbonyl Compounds in Electronic Cigarette Vapors-Effects of Nicotine Solvent and Battery Output Voltage. Nicotine Tob. Res., 16: 1319$26,2014$.

7- Scheffler, S., Dieken, H., Krischenowski, O., Förster, C., Branscheid, D. and Aufderheide, M.: Evaluation of E-Cigarette Liquid Vapor and Mainstream Cigarette Smoke after Direct Exposure of Primary Human Bronchial Epithelial Cells. Int. J. Environ. Res. Public Health, 12: 3915-3925, 2015 . 
8- Bertholon, J., Becquemin, M., Roy, M., Roy, F., Ledur, D., Annesi Maesano, I. and Dautzenberg, B.: Comparison of the aerosol produced by electronic cigarettes with conventional cigarettes and the shisha. Rev. Mal. Respir., 30: 752-757, 2013.

9- Cressey, D.: E-cigarettes affect cells. Nature, 508: 159, 2014.

10- Bertholon, J., Becquemin, M., Annesi-Maesano, I. and Dautzenberg, B.: Electronic cigarettes: a short review. Respiration, 86:433-438, 2013.

11- Thomas, E., Gajghate, S., Rajesh, K., Ma, J., Kim, J., Sudini, K., Consolini, N., Stephania, A., Lomnicki, S., Hasan, F., Pekosz, A. and Biswal, S.: Exposure to Electronic Cigarettes Impairs Pulmonary Anti-Bacterial and Anti-Viral Defenses in a Mouse Model. PLOS. One, 10: e0116861, 2015.

12- Wu, Q., Jiang, D., Minor, M. and Chu, H.: Electronic Cigarette Liquid Increases Inflammation and Virus Infection in Primary Human Airway Epithelial Cells. PLOS. One, 9: e108342, 2014

13- Avsar, A., Darca, O., Topaloglu, B. and Bek, Y.: Association of passive smoking with caries and related salivary biomarkersin young children. Arch. Oral. Biol., 10: 969$74,2008$.

14- Ferragut, J., Cunha, M., Carvalho, C., Isayama, R. and Caldeira, E.: Epithelial-stromal interactions in salivary glands of rats exposed to chronic passive smoking. Archives of Oral Biology, 56, 580-587, 2011.

15- OECD Guide Lines For The Testing Of Chemicals, Subacute Inhalation Toxicity Testing, Original Test Guideline No 412, Environment Directorate, OECD, Paris, 2009.

16- OECD Guide Lines For The Testing Of Chemicals, Subchronic Inhalation Toxicity Testing, Original Test Guideline No 413, Environment Directorate, OECD, Paris, 2009.

17- Hayat, M.: Basic Technique for Transmission electron microscopy, 1st ed., Academic Press; Inc. Florida: p. 1-10, 1986.

18- Neville, B. and Day, T.: Oral cancer and precancerous lesions. Cancer J. Clin., 52: 195-215, 2002.

19- Ayers, J., Ribisl, K. and Brownstein, J.: Tracking the rise in popularity of electronic nicotine delivery systems (electronic cigarettes) using search query surveillance. Am. J. Prev. Med., 40: 448-453, 2011.
20- Etter, J.: Levels of saliva cotinine in electronic cigarette users. Addiction, 109: 825-829, 2014.

21- Callahan-Lyon, P.: Electronic cigarettes: human health effects. Tob. Control, 23:ii36-ii40, 2014.

22- Nowakowski, R.: Centers for Disease Control and Prevention. Ingestion of cigarettes and cigarette butts by Rhode Island children. MMWR, 46:125-128, 1997.

23- Binsawad, A., Abdul Hakeem, M., ElQudsi, F., Ameen, H. and El Karium, S.: The possible protective role of antioxidants (selenium, vitamin E) in reducing smoking effects on testis of albino rats. Ass. Univ. Bull. Environ. Res., 14: 61-76, 2011.

24- Lina, S., Hashida, N. and Eliza, H..: Role of Habbatus sauda towards the histological features of nicotine treated male rats seminal vesicle and prostate gland. Biomedical Research, 25: 11-18, 2014.

25- Valenca, S., Gouveia, L., Pimenta, W. and Porto, L.: Effects of Oral Nicotine on Rat Liver Stereology. Int. J. Morphol., 26:1013-1022, 2008.

26- Gawish, A., Issa A., Bassily, N. and Manaa, S.: Role of green tea on nicotine toxicity on liver and lung of mice: Histological and morphometrical studies. Afr. J. Biotechnol., 11: 2013-2025, 2012.

27- Cunha, F., Amorim Júnior, A., Amorim, M., Oliveira, E. and Silva Júnior, V.: Histopathological study of lingual circumvallate papillae and associated tissues in brazilian smoker and non-smoker individuals. J. Morphol. Sci., 27: 152-154, 2010.

28- Singha, S. and Kazi, A.: Histological Effects of Smokeless Tobacco onthe Endometrial Glandsof the Orally Treated Female Swiss Albino Rats. International Journal of Research in Science, 1: 24-26, 2015.

29- Bao, H., Vepakomma, M. and Sarkar, M.: Benzo(a)pyrene exposure induces CYP1A1 activity and expression in human endometrial cells. J. Steroid Biochem. Mol, Biol., 81:37-45, 2002.

30- Williams, M., Villarreal, A., Bozhilov, K., Lin, S. and Talbot, P.: Metal and silicate particles including nanoparticles are present in electronic cigarette cartomizer fluid and aerosol. PLOS ONE 8, e57987, 2013.

31- El-Meligy, M., Abdel Hady, R., Abdel Samaei, A. and Saad Eldien, H.: Effect of nicotine administration and its withdrawal on the reproductive organs, fertility and pregnancy outcome in female rats. Mansoura J. Forensic Med. Clin. Toxicol., 15: 95-132, 2007. 
32- Iranloye, B. and Bolarinwa, A.: Effect of nicotine administration on weight and histology of some vital visceral organs in female albino rats. Nigerian Journal of Physiological Sciences 24: 7-12, 2009.

33- Fujinami, Y., Fukui, T., Nakano, K., Ara, T., Fujigaki, Y., Imamura, Y ., Hattori, T ., Yanagisawa, S ., Kawakami, T. and Wang, P.: The effects of cigarette exposure on rat salivary proteins and salivary glands. Oral Diseases, 15 : 466-471, 2009.

34- Neogy, S., Mahapatra, S., Mandal, S. and Somenath, R.: Amelioratory effect of Andrographis paniculata Nees on liver, kidney, heart, lung and spleen during nicotine induced oxidative stress. Environ. Toxicol. Pharma., 25: 321-328, 2008.

35- McCord, J.: Human disease, free radicals, and the oxidant/ antioxidant balance. Clin. Biochem. 26, 351-357, 1993.

36- Aoshiba, K. and Nagai, A.: Oxidative stress, cell death, and other damage to alveolar epithelial cells induced by cigarette smoke. Tobacco Induced Dis., 1: 219-226, 2003.

37- Dhouib, H., Jallouli, M., Draief, M., El-Fazaa, S. and Bouraoui, S.: The Effect of Nicotine and its Interaction with Ethanol on Biochemical Parameters, Oxidative Damage and Histological Changes in the Rat's Liver. IOSRJESTFT., 8: 72-82, 2014.

38- Dianzani, M., Lipid peroxidation in ethanol poisoning: a critical reconsideration. Alcohol. 20: 161-173, 1985.

39- Gawish, A., Ramadan, S., Hassan, A. and Issa, A.: Morphometrical, Histopathological, and Cytogenetical Ameliorating Effects of Green Tea Extract on Nicotine Toxicity of the Testis of Rats. J Cytol. Histol., 1: 1-7, 2010.

40- Cervellati, F., Muresan, X., Sticozzi, C., Gambari, R., Montagner, G., Forman, H., Torricelli, C., Maioli, E. and Valacchi, G.: comparative effect between electronic and cigarette smoke in human keratinocytes and epithelial lung cells. Toxicol. In Vitro, 28: 999-1005, 2014.

41- Toyokuni, S.: Reactive oxygen species-induced molecular damage and its application in pathology. Pathol. Int., 12: 49-61, 1999.

42- Yamazaki, H., Inoue, K., Hashimoto, M. and Shimada, T.: Role of CYP2A6 and CYP2B6 in nicotine C-oxidation by human liver microsomes. Arch. Toxicol., 73: 65-70, 1999. 